\title{
Pseudospectral Method Based on Müntz-Legendre Wavelets for Solving the Abel Integral Equation
}

\author{
Ioannis Dassios, ${ }^{1}$ Fairouz Tchier $\mathbb{D}^{2},{ }^{2}$ and F. M. O. Tawfiq ${ }^{2}$ \\ ${ }^{1}$ AMPSAS, University College Dublin, Dublin 4, Ireland \\ ${ }^{2}$ Department of Mathematics, King Saud University, P.O. Box 22452, Riyadh 11495, Saudi Arabia
}

Correspondence should be addressed to Fairouz Tchier; ftchier@ksu.edu.sa

Received 17 December 2021; Accepted 14 February 2022; Published 4 March 2022

Academic Editor: Azhar Hussain

Copyright (C) 2022 Ioannis Dassios et al. This is an open access article distributed under the Creative Commons Attribution License, which permits unrestricted use, distribution, and reproduction in any medium, provided the original work is properly cited.

\begin{abstract}
This paper deals with the numerical solution of the Abel integral equation based on Müntz-Legendre wavelets. To this end, the Abel integral operator is represented by Müntz-Legendre wavelets as an operational matrix. To find this matrix, we use the similarity between the Abel integral operator and the fractional integral operator. The proposed method can be easily used to solve weakly singular Volterra integral equations. We have proved the convergence of the proposed method. To demonstrate the ability and accuracy of the method, some numerical examples are presented.
\end{abstract}

\section{Introduction}

In this paper, we focus our attention on constructing and applying Müntz-Legendre (M-L) wavelets that will be used as the basis in the pseudospectral method to solve the famous Abel integral equation

$$
u(x)-\mathscr{A}^{\alpha}(k)(x)=f(x),
$$

in which Abel's integral operator $\mathscr{A}^{\alpha}(k)(x)$ of order $0<\alpha<1$ is defined in [1] as follows:

$\mathscr{A}^{\alpha}(k)(x):=\int_{0}^{x} k(x, s, u(s))(x-s)^{-\alpha} \mathrm{d} x, \quad x \in[0,1]$.

Here, given $\Omega=[0,1], f(x)$, and $k(x, s, u(s))$ are assumed to be continuous functions on $\Omega$ and $S$ with $S=\{(x, s, u): x, s \in[0,1], u \in \mathbb{R}\}$. Further, we suppose that the kernel function $k(x, s, u(s))$ is equal to the form $g(x, s) u(s)$. In other words, the desired equation is assumed to be linear.

The Abel equation is a special case of the integral equations with the weakly singular kernel that was first introduced by Abel. In investigating the generalization of the tautochrone problem, he introduced this equation [2]. This equation appears widely in modeling many physical problems, such as nuclear physics, X-ray radiography, fluid flow [3], scattering theory, plasma diagnostics, semiconductors, physical electronics, and nonlinear diffusion $[1,4]$. Given this equation's wide application, solving this equation is very important. But one cannot always solve the equation analytically, and we need to use numerical methods for it.

Among the many papers that have considered the numerical solution of this equation, we can mention some of them. Saadatmandi and Dehghan [5] utilized the collocation method based on shifted Legendre polynomials. Piessens and Verbaeten [6] introduced a numerical method based on Chebyshev polynomials, and after approximating the unknown solution based on these bases, they obtained the solution as a sum of hypergeometric functions. Using the Bernstein operational matrix, Singh et al. [7] introduced a stable numerical method to solve this problem. In [8], we can find the integrable solution of the Abel integral equation under certain conditions, and also the sufficient and necessary conditions for the existence of this solution are presented. In [9], the authors proposed the Laplace transform method to solve the problem, where they assumed that the solution would be differentiable and continuous. Saray [10] introduced a novel and efficient method based on 
Alpert's multiwavelets. In this work, after introducing the sparse representation of the Abel integral operator, the Abel integral equation is reduced to a sparse system of linear algebraic equations in the linear form, and this causes a reduction in time and computational costs. In [11], the unbounded solutions of the nonlinear Abel integral equations are investigated. Li and Zhao [12] used Mikusinski's operator of fractional order to solve the Abel integral equation.

The outline of this article is as follows: the M-L wavelets are constructed in Section 2, and then the Abel integral operator is represented based on these bases. In Section 3, the Abel integral equation is solved by using the pseudospectral method based on M-L wavelets. This section contains the error analysis and the conditions for convergence are investigated. To demonstrate the efficiency and accuracy of the presented method, some numerical examples are given in Section 4.

\section{Müntz-Legendre Wavelets}

As we know, multiresolution analysis (MRA) is a significant procedure for constructing wavelets. According to MRA, a family of nested subspaces exists such that they satisfy certain circumstances [13]

$$
\{0\} \subset \cdots \subset V_{-1} \subset V_{0} \subset V_{1} \subset \cdots \subset L^{2}(\Lambda),
$$

where $\Lambda$ is equal to $\mathbb{R}$ or any bounded interval.

Recently, the M-L wavelets have been used to solve some equations, such as fractional optimal control problems [14], fractional pantograph differential equations [15], fractional differential equations [16], and multiorder fractional differential equations [17]. To solve the Abel equation, we first briefly introduce the M-L wavelets as follows.

Given $J \in \mathbb{N}_{0}$, assume that the subspace $V_{J} \in L^{2}(\Lambda)$ is spanned by the scaled and translated version of a set of bases, which are called multiscaling functions, viz.,

$$
V_{J}=\left\{\operatorname{span}\left\{\phi_{J, b}^{n}: b \in \mathscr{B}, n \in \mathscr{R}\right\}\right\},
$$

where $\mathscr{B}:=\left\{0,1, \ldots, 2^{J}-1\right\}$ and $\mathscr{R}:=\{0,1, \ldots, r-1\}$ with $r \in \mathbb{N}$. The parameter $J$ is called refinement level and $r$ is the multiplicity parameter. In the sequel, we intend to introduce the functions $\phi_{J, b}^{n}$.

Motivated by [17], we denote the M-L polynomials $L_{n}(x)$ as

$$
L_{n}(x)=\sum_{k=0}^{n} l_{k, n} x^{\lambda_{k}}, \quad x \in \Omega,
$$

where $\lambda_{k}:=\{k \mu: \mu \in \mathbb{R}, k=0, \ldots, n\}$ and the coefficient $l_{k, n}$ is obtained by

$$
l_{k, n}:=\frac{\prod_{i=0}^{n-1}\left(\lambda_{k}+\lambda_{i}+1\right)}{\prod_{i=0, i \neq k}^{n}\left(\lambda_{k}-\lambda_{i}\right) .}
$$

It can be easily shown that these polynomials satisfy the orthogonality requirements and form an orthogonal system, via

$$
\left\langle L_{n}(x), L_{n^{\prime}}(x)\right\rangle=\int_{0}^{1} L_{n}(x) L_{n^{\prime}}(x) \mathrm{d} x=\frac{\delta_{n^{\prime}, n}}{2 \lambda_{n}+1}, \quad n \geq n^{\prime},
$$

where $\delta_{m_{l}, m}$ denotes the Kronecker symbol and is given by

$$
\delta_{n^{\prime}, n}:= \begin{cases}1, & n^{\prime}=n, \\ 0, & n \geq n^{\prime} .\end{cases}
$$

Considering the definition of $L_{n}(x)$, one can introduce the M-L wavelets [17], via

$$
\phi_{J, b}^{n}= \begin{cases}2^{J / 2} \sqrt{2 \lambda_{n}+1} L_{n}\left(2^{J} x-b\right), & \frac{b}{2^{J}} \leq x \leq \frac{b+1}{2^{J}}, \\ 0, & \text { otherwise. }\end{cases}
$$

Due to the definition of M-L wavelets, one can introduce the projection operator $\mathscr{P}$ that maps any function $u \in L^{2}(\Omega)$ onto $V_{J}$ as follows:

$$
u(x) \approx \mathscr{P}(u)(x)=\sum_{b=0}^{2^{J}-1} \sum_{n=0}^{r-1} u_{b, n} \phi_{J, b}^{n}(x)=U^{T} \Phi(x),
$$

where and throughout the paper, the superscript $T$ is used for the matrix transpose. Here $\Phi(x)$ is a vector function of dimension $N=2^{J} r$ whose $(b r+n+1)$-th element is $\phi_{J, b}^{n}(x)$, and the $(b r+n+1)$-th element of the vector $U$ is evaluated by

$$
u_{b, n}=\left\langle u, \phi_{J, b}^{n}\right\rangle=\int_{0}^{1} u(x) \phi_{J, b}^{n}(x) \mathrm{d} x .
$$

It follows from [15] that one may be able to bound the projection error $\mathscr{P}$ in the sense of Sobolev norms.

Lemma 1 (see [15]). Given $n \geq 0$, assume that $r>m$. If $u \in H^{m}(\Omega)$, then

$$
\|u-\mathscr{P}(u)\|_{L_{2}(\Omega)} \leq c(r-1)^{-m}\left(2^{J-1}\right)^{-m}\left\|u^{(m)}\right\|_{L_{2}(\Omega)^{\prime}}
$$

and for $s \geq 1$, we have

$\|u-\mathscr{P}(u)\|_{H^{s}(\Omega)} \leq c(r-1)^{2 s-(1 / 2)-m}\left(2^{J-1}\right)^{s-m}\left\|u^{(m)}\right\|_{L_{2}(\Omega)}$

in which $H^{m}(\Omega)$ is the Sobolev space and the related norm is determined by

$$
\|u\|_{H^{m}(\Omega)}=\left(\sum_{j=0}^{m}\left\|u^{(j)}\right\|_{L_{2}(\Omega)}^{2}\right)^{1 / 2} .
$$

2.1. Representation of Abel Integral Operator in Müntz-Legendre Wavelets. In this subsection, we consider the Abel operator as a fractional integral operator, and after representing the fractional integral operator in M-L wavelets as an operational matrix, we find a representation of the Abel 
integral operator in M-L wavelets. To this end, it is necessary to define some concepts about fraction calculation.

Definition 1 (see [18]). Let $u \in L_{1}[a, b]$. The Riemann-Liouville fractional integral operator $\mathscr{F}_{a}^{\alpha}$ of order $\alpha \in \mathbb{R}^{+}$is determined by

$\mathcal{F}_{a}^{\alpha}(u)(x):=\frac{1}{\Gamma(\alpha)} \int_{a}^{x}(x-s)^{\alpha-1} u(s) \mathrm{d} s, \quad x \in[a, b]$,

where $\Gamma(\alpha)$ represents the gamma function.

Remark 1. It can be verified that the fractional integral of the functions $x^{\kappa}$ is given by

$$
\mathscr{J}_{a}^{\alpha}\left(x^{\kappa}\right)=\frac{\Gamma(\kappa+1)}{\Gamma(\kappa+\alpha+1)} x^{\kappa+\alpha} .
$$

Lemma 2 (cf Lemma 2.1(a). see [19]). The fractional integration operators $\mathscr{F}_{a}^{\alpha}$ are bound in $L^{p}([a, b])$ for $1 \leq p \leq \infty$ as follows:

$$
\left\|\mathcal{F}_{a}^{\alpha}(u)\right\|_{p} \leq K\|u\|_{p}, \quad K:=\frac{(b-a)^{\alpha}}{\Gamma(\alpha+1)} .
$$

It follows from [19] that if $u \in L_{1}[a, b]$, then the function $\mathcal{F}_{a}^{\alpha} u$ itself belongs to $L_{1}[a, b]$. We recall that the Abel integral operator of order $\alpha \in(0,1)$ is determined by

$$
\mathscr{A}^{\alpha}(u)(x)=\int_{0}^{x}(x-s)^{-\alpha} u(s) \mathrm{d} s .
$$

There is a similarity between the Abel integral operator $\mathscr{A}^{\alpha}$ and the Riemann-Liouville fractional integral operator $\mathcal{F}_{0}^{\beta}(\beta:=1-\alpha)$, viz,

$$
\mathscr{A}^{\alpha}=\Gamma(\alpha) \mathscr{F}_{a}^{1-\alpha} .
$$

Thus, we can use the Riemann-Liouville fractional integral operator $\mathscr{F}_{0}^{\beta}$ instead of the Abel integral operator $\mathscr{A}^{\alpha}$.

According to the definition of M-L wavelets, the fractional integral operator $\mathcal{F}_{0}^{\beta}$ acting on the vector function $\Phi(x)$ may be written as an expansion of M-L wavelets $\Phi(x)$, i.e.,

$$
\mathscr{P}\left(\mathscr{J}_{0}^{\beta}\right)(\Phi(x))=I_{\beta}(x) \Phi(x), \quad \beta \in(0,1),
$$

where $I_{\beta}(x)$ is an $N \times N$ matrix and is famous as the operational matrix of fractional integral for the M-L wavelets.

Before we look at how to calculate the elements of the aforementioned matrix, it is intransitive to introduce the piecewise fractional-order Taylor functions. Let $J \in \mathbb{Z}^{+} \cup\{0\}$ be a fixed number, the piecewise fractional-order Taylor functions can be defined in the following form:

$$
\psi_{J, b}^{n}= \begin{cases}t^{\lambda_{n}}, & \frac{b}{2^{J}} \leq x \leq \frac{b+1}{2^{J}}, \\ 0, & \text { otherwise. }\end{cases}
$$

By introducing the square matrix $T$ of dimension $N \times N$, whose $(i, j)$-th element is computed by

$$
\begin{array}{r}
T_{i, j}=\left\langle\Phi_{i}(x), \Psi_{j}(x)\right\rangle=\int_{0}^{1} \Phi_{i}(x), \Psi_{j}(x) \mathrm{d} x, \\
i, j=1, \ldots, N .
\end{array}
$$

We can expand any elements of vector function $\Phi(x)$ (M-L wavelets) by the piecewise fractional-order Taylor functions $\Psi(x)$, viz,

$$
\Phi(x)=T^{-1} \Psi(x) .
$$

Here the matrix $T$ is a $N \times N$ matrix and it is called the transformation matrix. In the sequel, we assume that $Q$ is a vector of dimension $r$ whose $i$-th element is $x^{\lambda_{i}}$. Thus, it is easy to show that

$$
\Psi(x)=[Q, \ldots, Q]^{T} .
$$

It follows from (16) that one can find the $i$-th element of $\mathscr{F}_{0}^{\beta}(\Psi)(x)$, via

$$
\mathscr{F}_{0}^{\beta}\left(\Psi_{i}\right)(x)=\frac{\Gamma\left(\lambda_{i}+1\right)}{\Gamma\left(\lambda_{i}+\beta+1\right)} x^{\lambda_{i}+\beta} .
$$

This gives rise to introduce a diagonal matrix $I_{\Phi, \beta}(x)$, such that

$$
\mathscr{F}_{0}^{\beta}(\Psi)(x)=I_{\Psi, \beta}(x) \Psi(x) .
$$

It is worth noting that this matrix expresses the fractional integral of functions $\Psi$ as a combination of themselves and has the following form:

$$
I_{\Psi, \beta}(x)=\operatorname{diag}\left[P_{\beta}(x), \ldots, P_{\beta}(x)\right],
$$

where $P_{\beta}(x):=x^{\beta} H\left(\mathscr{F}_{0}^{\beta}(Q)(x)=P_{\beta}(x) Q(x)\right)$ and $H$ is a diagonal matrix of the form

$$
(H)_{i, i}=\frac{\Gamma\left(\lambda_{i}+1\right)}{\Gamma\left(\lambda_{i}+\beta+1\right)} .
$$

Now, we are able to introduce the operational matrix of fractional integral for the M-L wavelets via

$$
\begin{aligned}
\mathscr{P}_{J}\left(\mathcal{F}_{0}^{\beta}\right)(\Phi(x)) & =\mathscr{P}_{J}\left(\mathscr{F}_{0}^{\beta}\right)\left(T^{-1} \Psi(x)\right) \\
& =T^{-1} I_{\Psi, \beta}(x) \Psi(x)=T^{-1} I_{\Psi, \beta}(x) T \Phi(x) .
\end{aligned}
$$

Thus, we get

$$
I_{\beta}(x):=T^{-1} I_{\Psi, \beta}(x) T .
$$

\section{Pseudospectral Method}

To derive the numerical solution of the second kind of Abel integral equation based on the pseudospectral method, we can approximate the unknown solution with the projection operator $\mathscr{P}_{J}$, as follows:

$$
u \approx \mathscr{P}_{J}(u)=U^{T} \Psi,
$$

where $U$ is a vector of dimension $N$, whose elements should be found. Note that the function $f(x)$ and the kernel 
function $k\left(x, s, \mathscr{P}_{J}(u)(s)\right)$ can be approximated in the same manner, i.e.,

$$
\begin{aligned}
f(x) & \approx \mathscr{P}_{J}(u)=F^{T} \Psi, \\
k\left(x, s, \mathscr{P}_{J}(u)(s)\right) & \approx \mathscr{P}_{J}(k)\left(x, s, \mathscr{P}_{J}(u)(s)\right) \\
& =\Psi^{T}(x) K \Psi(s),
\end{aligned}
$$

where $F \in \mathbb{R}^{N}$ and $K \in \mathbb{R}^{N \times N}$. By substituting (32) into the integral part of the Abel integral equation (1), we obtain

$$
\begin{aligned}
& \mathscr{P}_{J}\left(\Psi(x)^{T} \widetilde{K} \int_{0}^{x}(x-s)^{-\alpha} \Psi(s) \mathrm{d} s\right)=\mathscr{P}_{J}^{r}\left(\Psi(x)^{T} \widetilde{K} I_{\alpha} \Psi(x)\right) \\
& =\widehat{K} \Psi(x) .
\end{aligned}
$$

We now substitute equations (31)-(33) into Abel integral equation (1) and simplify to get

$$
r(x):=(U-F+\widehat{K})^{T} \Psi(x)=0,
$$

where $r(x)$ is the residual function that our goal is to reduce to zero. Let $\left\{x_{i}\right\}$ be a number of points in $\Omega$, we select the solution that satisfies the collocation condition $r\left(x_{i}\right)=0$, where $\left\{x_{i}\right\}$ are called the collocation points. In this paper, we use the shifted Chebyshev and Legendre polynomials zeros as collocation points. The collocation method gives rise to a system of linear or nonlinear algebraic equations. One can derive the unknown coefficients $U$ after solving this system.

3.1. Error Analysis. We write the Abel integral equation (1) in the form

$$
(I-\mathscr{K}) u=f
$$

where $\mathscr{K}$ is a compact operator that maps any continuous function onto $C[0,1]$. As we said, our goal is to reduce the residual function $r(x)$ to zero. Symbolically, we have

$$
r(x)=(I-\mathscr{K}) u_{J}-f,
$$

where $u_{J}:=\mathscr{P}_{J}(u)$. We note that $\mathscr{P}_{J}(r)(x)=0$ if and only if $r\left(x_{i}\right)=0$ or equivalently,

$$
\mathscr{P}_{J}(I-\mathscr{K}) u_{J}=\mathscr{P}_{J}(f) .
$$

Let $u_{J}$ is a solution of (37), then by applying $\mathscr{P}_{J}\left(u_{J}\right)=u_{J}$, equation (37) can be written as follows:

$$
\left(I-\mathscr{P}_{J} \mathscr{K}\right) u_{J}=\mathscr{P}_{J}(f) .
$$

Since both the original equations (1) and (38) are defined on $C[0,1]$, then for the error analysis, we compare them.

Theorem 1. Let us assume that $I-\mathscr{K}: C[0,1] \longrightarrow C[0,1]$ is a bijections operator. Further, let us assume

$$
\left\|\mathscr{K}-\mathscr{P}_{J} \mathscr{K}\right\| \longrightarrow 0, \quad \text { as } r \longrightarrow \infty \text {. }
$$

Then for all sufficiently large $r(r \leq N)$, the operator $\left(I-\mathscr{P}_{J} \mathscr{K}\right)^{-1}$ exists as a bounded operator from $C[0,1]$ to $C[0,1]$. Moreover, it is uniformly bounded

$$
\sup _{r \leq N}\left\|\left(I-\mathscr{P}_{J} \mathscr{K}\right)^{-1}\right\|<\infty
$$

For the solutions of (35) and (38)

$$
\begin{aligned}
u-u_{J} & =\left(I-\mathscr{P}_{J} \mathscr{K}\right)^{-1}\left(u-\mathscr{P}_{J}(u)\right), \\
\frac{1}{\left\|I-\mathscr{P}_{J} \mathscr{K}\right\|}\left\|u-\mathscr{P}_{J}(u)\right\| & \leq\left\|u-u_{J}\right\| \\
& \leq\left\|\left(I-\mathscr{P}_{J} \mathscr{K}\right)^{-1}\right\|\left\|u-\mathscr{P}_{J}(u)\right\| .
\end{aligned}
$$

Proof. For details, refer to [20].

Since $\mathscr{K}: C[0,1] \longrightarrow C[0,1]$ is a compact operator and $\mathscr{P}_{J}$ is a bounded projection, such that $\mathscr{P}_{J} u \longrightarrow 0$ as $r \longrightarrow \infty$. Then motivated by [20] (Lemma 3.1.2), we have

$$
\left\|\mathscr{K}-\mathscr{P}_{J} \mathscr{K}\right\| \longrightarrow 0, \quad \text { as } r \longrightarrow \infty \text {. }
$$

Therefore, the condition of Theorem 1 is held.

\section{Numerical Examples}

In this section, some numerical examples are solved to show the validity and efficiency of the method. To do this, we carry out the Maple and MATLAB software simultaneously.

Example 1. For the first example, let us consider the linear Abel integral equation of the second kind with the kernel function $k(t, x, u(x)):=(1 / 10 \Gamma(1-\alpha)) u(x)$, and $f(t)=1$. The exact solution is given in [10] as follows:

$$
u(t)=E_{1-\alpha, 1}\left(\frac{t^{1-\alpha}}{10}\right)
$$

where $E_{\sigma, \beta}$ is the Mittag-Leffler function

$$
E_{\sigma, \beta}(z)=\sum_{l=0}^{\infty} \frac{z^{l}}{\Gamma(\sigma l+\beta)}, \quad \sigma, \beta, z \in \mathbb{R}, \sigma>0 .
$$

Table 1 shows the absolute value of errors at different times $x_{i}$ when the collocation points are chosen to be the Legendre polynomial nodes. As we expected, when the $r$ increases (the degree of the bases as well as the number of collocation points increases) the error will decrease. To show the effect of the parameter $\mu$ in the $L^{2}$-error, we plot Figure 1 and report Table 2. Figure 2 illustrates the effect of the choosing nodes on the $L^{2}$-error and also absolute value of errors at the Chebyshev nodes using different multiplicity $r$. Also, we can see the effect of the multiplicity parameter $r$ on the $L^{2}$-error and absolute error in Figure 2.

Example 2. The second example is devoted to the Abel integral equation (1) with

$$
\begin{aligned}
f(t) & :=2 \sqrt{t}, \\
k(t, x, u(x)) & :=u(x) .
\end{aligned}
$$


TABLE 1: The absolute errors at the Legendre nodes for Example 1.

\begin{tabular}{lccr}
\hline$x$ & $r=5$ & $r=7$ & $r=9$ \\
\hline 0.1 & $9.72 e-4$ & $2.78 e-4$ & $1.19 e-4$ \\
0.2 & $2.41 e-4$ & $2.24 e-4$ & $1.30 e-5$ \\
0.3 & $3.15 e-4$ & $1.22 e-6$ & $4.64 e-5$ \\
0.4 & $2.93 e-4$ & $1.09 e-4$ & $4.83 e-5$ \\
0.5 & $1.23 e-5$ & $7.38 e-7$ & $1.93 e-6$ \\
0.6 & $1.94 e-4$ & $7.95 e-6$ & $3.14 e-5$ \\
0.7 & $1.53 e-4$ & $6.80 e-6$ & $2.21 e-5$ \\
0.8 & $7.94 e-5$ & $6.32 e-5$ & $6.04 e-6$ \\
0.9 & $1.89 e-4$ & $4.44 e-5$ & $1.60 e-5$ \\
1.0 & $4.09 e-4$ & $1.60 e-4$ & $7.86 e-5$ \\
\hline
\end{tabular}

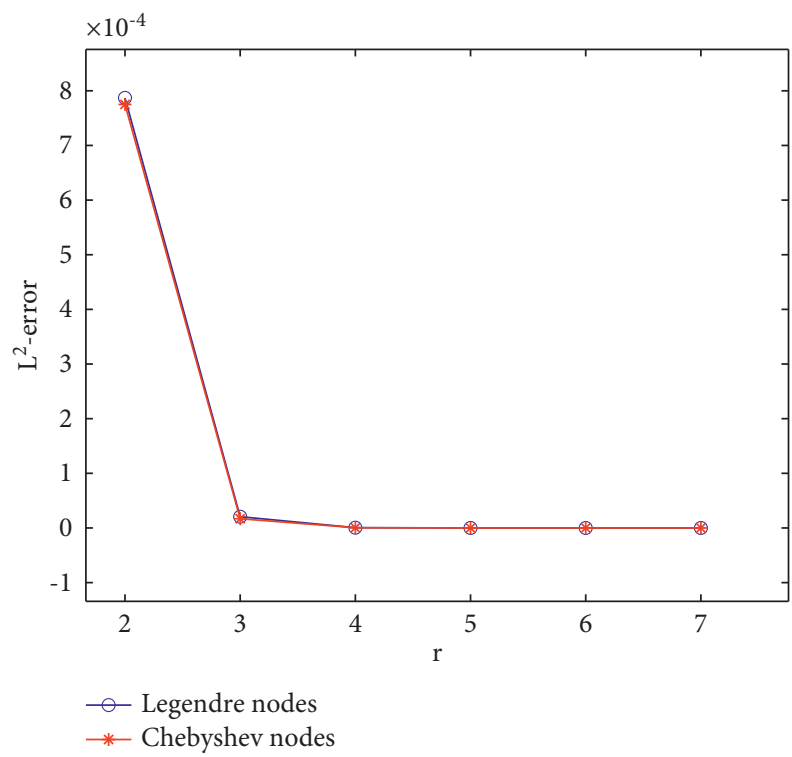

(a)

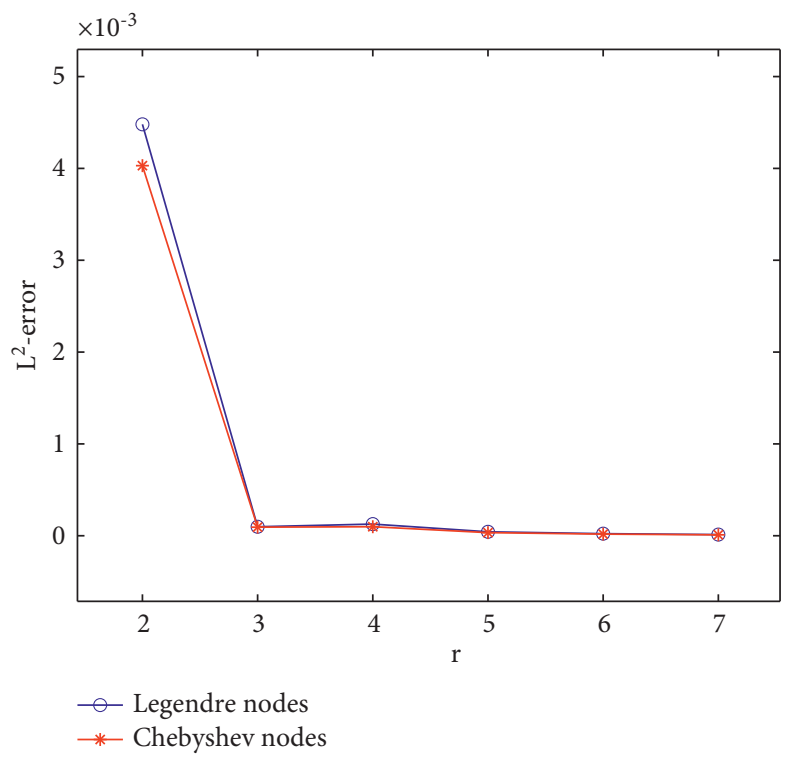

(b)

FIGURE 1: Plot of the $L^{2}$-errors taking $\mu=(1 / 2)$ (a) and $\mu=(1 / 3)$ (b) at the Legendre and Chebyshev nodes for Example 1.

TABle 2: The $L^{2}$-errors at the Legendre and Chebyshev nodes taking different $\mu$ for Example 1 .

\begin{tabular}{ccccc}
\hline$r$ & $\mu=(1 / 2)$ & & $\mu=(1 / 3)$ \\
& Chebyshev nodes & Legendre nodes & Chebyshev nodes & Legendre nodes \\
\hline 2 & $7.57 e-4$ & $7.87 e-4$ & $4.03 e-3$ & $4.48 e-3$ \\
3 & $1.69 e-5$ & $2.06 e-5$ & $9.34 e-5$ & $9.79 e-5$ \\
4 & $3.82 e-7$ & $5.32 e-7$ & $9.81 e-5$ & $1.27 e-4$ \\
5 & $8.58 e-9$ & $1.33 e-8$ & $3.37 e-5$ & $4.29 e-5$ \\
6 & $1.89 e-10$ & $3.16 e-10$ & $1.76 e-5$ & $2.26 e-5$ \\
7 & $4.06 e-12$ & $7.19 e-12$ & $9.89 e-6$ & $1.27 e-5$ \\
\hline
\end{tabular}
[4].

The exact solution is given by $u(t)=1-e^{\pi t} \operatorname{erf} c(\sqrt{\pi t})$

To show the effect of the multiplicity parameter $r$ and choosing the collocation points, we report Table 3. This Table also illustrates the effect of parameter $\mu$ on the $L^{2}$-error. Due to Table 3, it is obvious that these three parameters have a direct effect on the $L^{2}$-error such that when $r$ increases, the error decreases. Also, choosing the Chebyshev nodes gives us a better result than Legendre nodes. In Figure 3, we demonstrate the absolute error when multiplicity parameter $r$ increases taking $\mu=(1 / 2)$ and different collocation points.

Example 3. Let us consider the following Abel integral equation:

$$
f(t):=\sin (x)-\frac{4}{3} x^{3 / 2}{ }_{1} F_{2}\left(1 ; \frac{5}{4}, \frac{7}{4} ;-\frac{x^{2}}{4}\right), \quad k(t, x, u(x)):=u(x),
$$




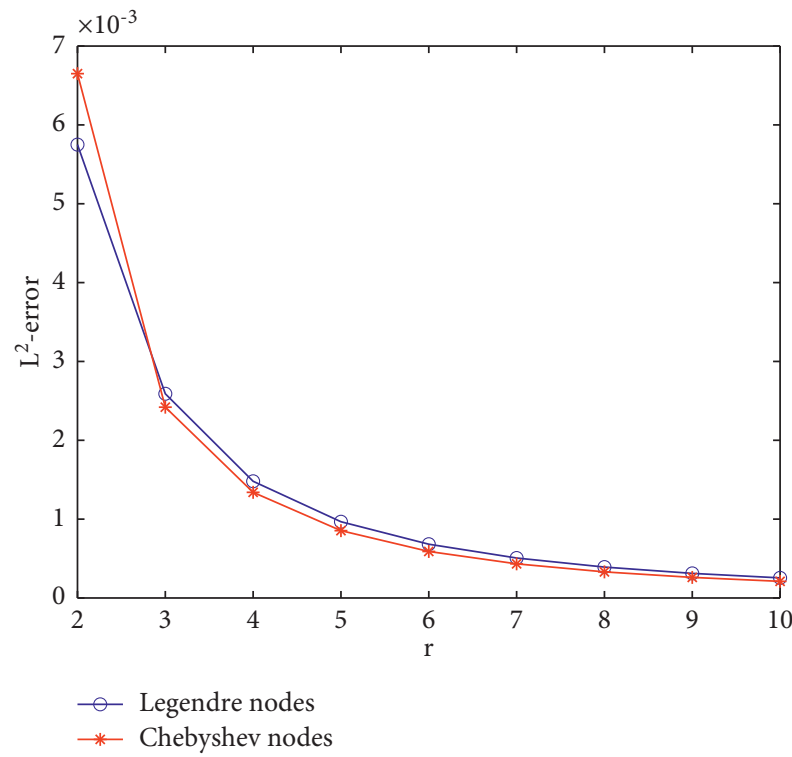

(a)

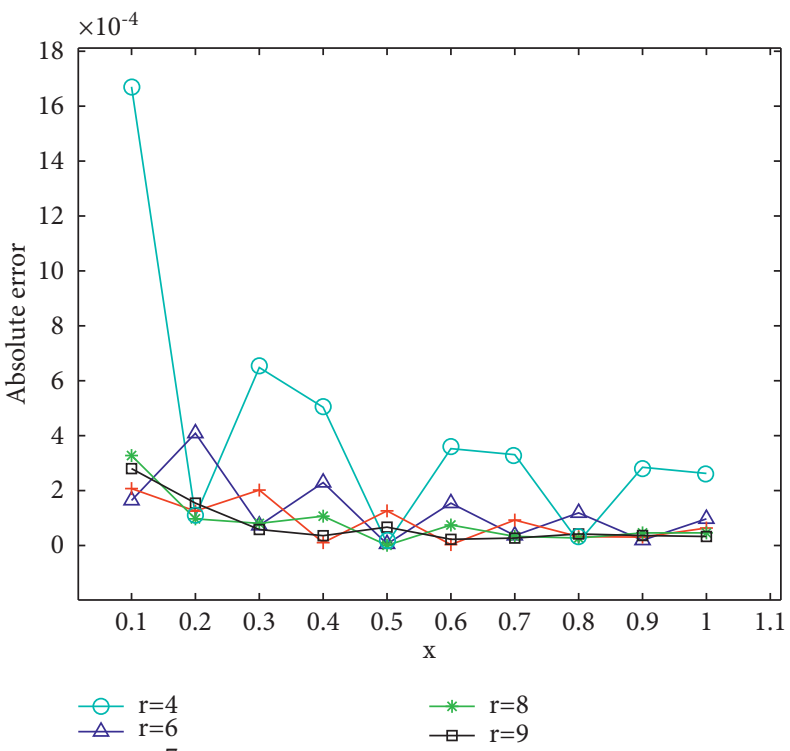

(b)

Figure 2: Plot of the $L^{2}$-errors (a) taking the Legendre and Chebyshev nodes and the absolute error at the Chebyshev nodes (b) for Example 1.

TABle 3: The $L^{2}$-errors at the Legendre and Chebyshev nodes taking different $\mu$ for Example 2.

\begin{tabular}{lcccc}
\hline$r$ & $\mu=(1 / 2)$ & & $\mu=1$ \\
& Chebyshev nodes & Legendre nodes & Chebyshev nodes & Legendre nodes \\
\hline 2 & $3.38 e-2$ & $3.52 e-2$ & $5.68 e-2$ & $5.62 e-2$ \\
3 & $9.21 e-2$ & $1.08 e-2$ & $3.15 e-2$ & $2.15 e-2$ \\
4 & $2.57 e-3$ & $3.36 e-3$ & $1.95 e-2$ & $2.00 e-2$ \\
5 & $7.29 e-4$ & $1.04 e-3$ & $1.31 e-2$ & $1.38 e-2$ \\
6 & $2.06 e-4$ & $3.17 e-4$ & $9.40 e-3$ & $7.01 e-2$ \\
7 & $5.77 e-5$ & $9.40 e-5$ & $7.04 e-3$ & $7.73 e-3$ \\
\hline
\end{tabular}

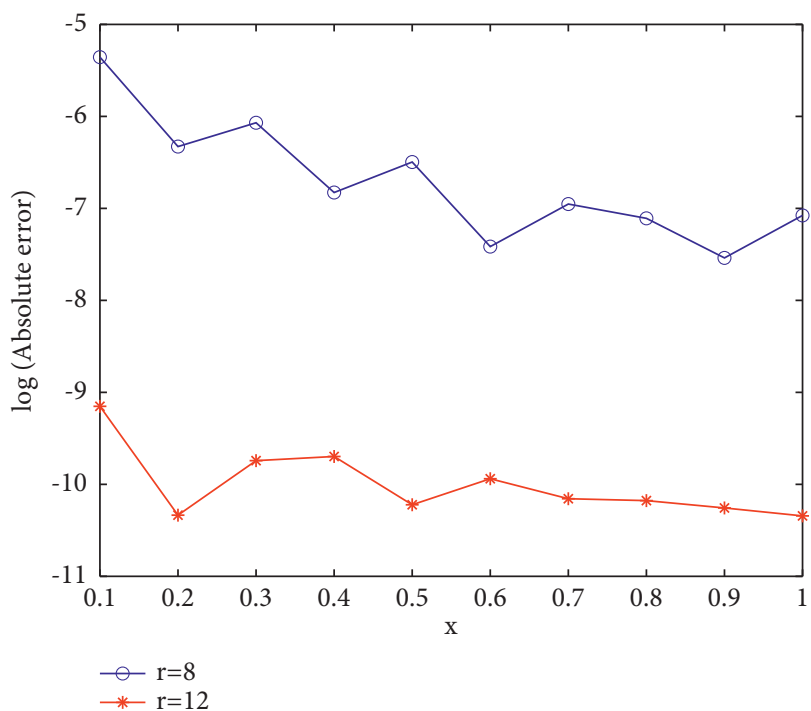

(a)

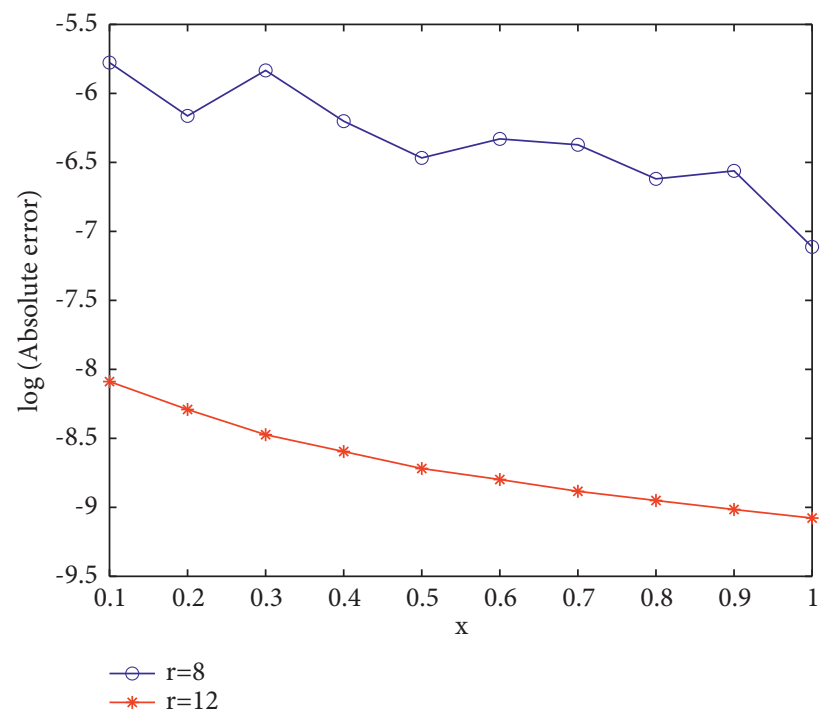

(b)

Figure 3: Plot of the absolute error at the Chebyshev nodes (a) and the Legendre nodes (b) taking different $r$ for Example 2. 
TABLE 4: The absolute errors at the Chebyshev nodes for Example 3.

\begin{tabular}{lccr}
\hline$x$ & $r=6$ & $r=8$ & $r=10$ \\
\hline 0.1 & $1.42 e-4$ & $1.20 e-6$ & $1.60 e-7$ \\
0.2 & $6.05 e-6$ & $2.45 e-6$ & $1.53 e-9$ \\
0.3 & $2.39 e-6$ & $3.44 e-7$ & $4.50 e-8$ \\
0.4 & $4.67 e-5$ & $6.55 e-7$ & $6.19 e-8$ \\
0.5 & $8.29 e-5$ & $1.61 e-6$ & $7.59 e-8$ \\
0.6 & $1.03 e-4$ & $1.87 e-6$ & $1.08 e-7$ \\
0.7 & $1.23 e-4$ & $2.31 e-6$ & $1.48 e-7$ \\
0.8 & $1.66 e-4$ & $3.36 e-6$ & $2.01 e-7$ \\
0.9 & $2.40 e-4$ & $4.64 e-6$ & $2.76 e-7$ \\
1.0 & $3.21 e-4$ & $6.34 e-6$ & $3.78 e-7$ \\
\hline
\end{tabular}

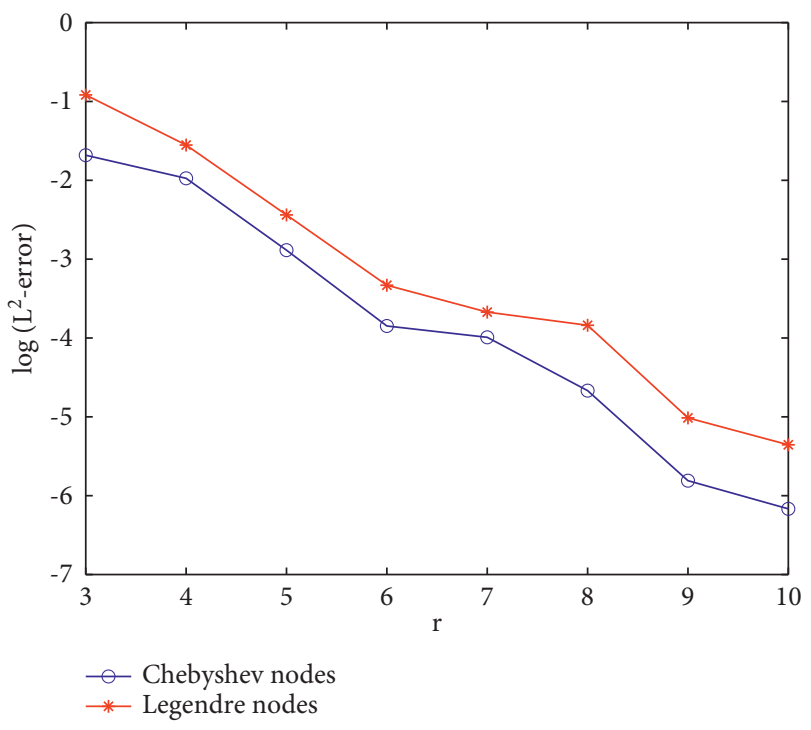

Figure 4: Plot of the $L^{2}$-errors taking $\mu=(1 / 2)$ at the Legendre and Chebyshev nodes for Example 3.

in which ${ }_{1} F_{2}$ is the hypergeometric function defined in [21]. Also, the exact solution is $u(x)=\sin (x)$.

Table 4 shows the absolute value of errors at different times $x_{i}$ when the collocation points are chosen to be the Chebyshev polynomials nodes. Figure 4 illustrates the effect of selecting the Chebyshev and Legendre nodes. We can also see the effect of increasing the parameter $r$. It is observed that by increasing the parameter $r$, the error decreases.

\section{Conclusion}

In this paper, we utilize an efficient algorithm based on the wavelet pseudospectral method to solve the well-known Abel integral equation. This method can easily be used to solve weakly singular Volterra integral equations, and this shows the ability of the proposed method. We have compared the method with other methods and shown that this method offers better results. We have proved the convergence of the proposed method. Given the construction of these bases and the role of the parameter $\mu$, which can be polynomials with fractional powers, compared to other bases, if the exact solution or the known functions in the equation are of the fractional type, the proposed method will provide better results.

\section{Data Availability}

The data used to support this study are included within this article.

\section{Conflicts of Interest}

The authors declare that there are no conflicts of interest with this work.

\section{Authors' Contributions}

All authors read and approved the final manuscript. All authors contributed equally and significantly to the writing of this paper. 


\section{Acknowledgments}

This work is supported by the Researchers Supporting Project Number (RSP-2021/401), King Saud University, Riyadh, Saudi Arabia.

\section{References}

[1] R. Gorenflo and S. Vessella, "Abel integral equations, analysis and applications," in Lecture Notes in MathematicsSpringer, Berlin, Germany, 1991.

[2] G. Simmons, Differential Equations with Applications and Historical Notes, McGraw-Hill, New York, NY, USA, 1972.

[3] J. Goncerzewicz, H. Marcinkowska, W. Okrasiński, and K. Tabisz, "On the percolation of water from a cylindrical reservoir into the surrounding soil," Applicationes Mathematicae, vol. 16, no. 2, pp. 249-261, 1978.

[4] A. M. Wazwaz, Linear and Nonlinear Integral Equations: Methods and Applications, Higher Education Press and Springer, Berlin, Germany, 2011.

[5] A. Saadatmandi and M. Dehghan, "A collocation method for solving Abel's integral equations of first and second kinds," Zeitschrift für Naturforschung A, vol. 63, no. 12, pp. 752-756, 2008.

[6] R. Piessens and P. Verbaeten, "Numerical solution of the abel integral equation," Bit, vol. 13, no. 4, pp. 451-457, 1973.

[7] O. P. Singh, V. K. Singh, and R. K. Pandey, "A stable numerical inversion of Abel's integral equation using almost Bernstein operational matrix," Journal of Quantitative Spectroscopy and Radiative Transfer, vol. 111, no. 1, pp. 245-252, 2010.

[8] J. D. Tamarkin, “On integrable solutions of Abel's integral equation," Annals of Mathematics, vol. 31, no. 2, pp. 219-229, 1930.

[9] G. Doetsch, "Integro-differentialgleichungen vom faltungstypus," Mathematische Annalen, vol. 89, 1923.

[10] B. N. Saray, "Abel's integral operator: sparse representation based on multiwavelets," BIT Numerical Mathematics, vol. 61, pp. 587-606, 2021.

[11] M. R. Arias, R. Benítez, and V. J. Bolós, “Attraction properties of unbounded solutions for a nonlinear Abel integral equation," Journal of Integral Equations and Applications, vol. 19, no. 4, pp. 439-452, 2007.

[12] M. Li and W. Zhao, "Solving Abel's type integral equation with Mikusinski's operator of fractional order," Advances in Mathematical Physics, vol. 2013, Article ID 806984, 4 pages, 2013.

[13] S. G. Mallat, A Wavelet Tour of Signal Processing, Academic Press, Cambridge, MA, USA, 1999.

[14] P. Rahimkhani and Y. Ordokhani, "Numerical solution a class of 2D fractional optimal control problems by using 2D Müntz-Legendre wavelets," Optimal Control Applications and Methods, vol. 39, no. 6, pp. 1916-1934, 2018.

[15] P. Rahimkhani, Y. Ordokhani, and E. Babolian, "MüntzLegendre wavelet operational matrix of fractional-order integration and its applications for solving the fractional pantograph differential equations," Numerical Algorithms, vol. 77, pp. 1283-1305, 2018.

[16] P. Mokhtary, F. Ghoreishi, and H. M. Srivastava, "The MüntzLegendre Tau method for fractional differential equations," Applied Mathematical Modelling, vol. 40, no. 2, pp. 671-684, 2016.

[17] H. B. Jebreen and F. Tchier, "A new scheme for solving multiorder fractional differential equations based on Müntz- legendre wavelets," Complexity, vol. 2021, Article ID 9915551, 9 pages, 2021.

[18] A. Afarideh, F. Dastmalchi Saei, M. Lakestani, and B. Nemati Saray, "Pseudospectral method for solving fractional SturmLiouville problem using Chebyshev cardinal functions," Physica Scripta, vol. 96, no. 12, Article ID 125267, 2021.

[19] A. Kilbas, H. M. Srivastava, and J. J. Trujillo, Theory and Applications of Fractional Differential Equations, Vol. 24, Elsevier, Amsterdam, Netherlands, 2006.

[20] K. E. Atkinson, The Numerical Solution of Integral Equations of the Second Kind, Cambridge University Press, Cambridge, UK, 1997.

[21] R. Beals and R. Wong, Special Functions, Cambridge University Press, Cambridge, UK, 2010. 